Bulletin of the Section of Logic

Volume 51/1 (2022), pp. 27-56

https://doi.org/10.18778/0138-0680.2021.16

\title{
TERNARY RELATIONAL SEMANTICS FOR THE VARIANTS OF BN4 AND E4 WHICH CONTAIN ROUTLEY AND MEYER'S LOGIC B
}

\begin{abstract}
Six interesting variants of the logics BN4 and E4-which can be considered as the 4-valued logics of the relevant conditional and (relevant) entailment, respectivelywere previously developed in the literature. All these systems are related to the family of relevant logics and contain Routley and Meyer's basic logic B, which is well-known to be specifically associated with the ternary relational semantics. The aim of this paper is to develop reduced general Routley-Meyer semantics for them. Strong soundness and completeness theorems are proved for each one of the logics.

Keywords: Ternary relational semantics, relevant logics, 4-valued logics, Routley and Meyer's logic B, 4-valued quasi-relevant logics.
\end{abstract}

\section{Introduction}

Brady defined in 1982 the system BN4 (cf. [3]), a logic built upon the matrix MBN4. This matrix is the result of a modification of the function $f_{\rightarrow}$ for the conditional in Smiley's matrix MSm4, which is in its turn a simplification of a matrix which has played an important role in the development of relevant logics (cf. [11, pp. 176, ff.]), i.e., Anderson and Belnap's 8-element

${ }^{*}$ I would like to sincerely thank José Manuel Méndez for useful comments on a draft version of this paper.

Presented by: Norihiro Kamide

Received: November 7, 2020

Published online: September 2, 2021

(C) Copyright by Author(s), Łódź 2022

(C) Copyright for this edition by Uniwersytet Łódzki, Łódź 2022 
matrix $\mathrm{M}_{0}$ (cf. [1]). Smiley's matrix MSm4 is also the matrix characteristic of Anderson and Belnap's First Degree Entailment Logic (FDE; cf. [1, pp. 161-162]). The logic FDE is a well-known core non-classical system among many-valued and relevant logics (about its importance, cf. [8] and references therein) and is equivalent to Belnap and Dunn's logic B4 [2]. It is worth to mention here that, according to Slaney [12, p. 289], BN4 has the truth-functional implication most naturally associated the logic FDE. As a matter of fact, the logic BN4 finds an appealing place in the intersection between 4 -valued logics and members in the family of relevant logics. For instance, Meyer et al. maintain that "BN4 is the correct logic for the 4 -valued situation where the extra values are to be interpreted in the both and neither senses" [7, p. 253]. Accordingly, BN4 can also be seen as an interesting 4-valued extension of Routley and Meyer's basic logic B (cf. [11, chapter 4]) - a central system in the family of relevant logics whose significance is briefly explained below - since the logic BN4 was first developed by taking as the starting point the axiomatization of B, as Brady himself stated. As a matter of fact, even though it is tempting to read BN4 as the $\mathrm{B}$ (oth) and N(either) 4-valued logic, the label was chosen by Brady because "the system contains the basic system B of Routley et al. 1982, Chapter 4 , and has a characteristic 4-valued matrix set, one of the values being 'n', representing neither truth nor falsity" (cf. [3, p. 32, note 1]).

Routley and Meyer's relational semantics (R-M semantics) was introduced by the named authors in the early seventies of the past century to model relevant logics, but it was soon noticed to be a highly malleable instrument able of modelling other families of logics. The minimal (nonpositive) logic characterized by Routley-Meyer semantics is Sylvan and Plumwood's logic BM [13], which is in fact the result of dropping the double negation axioms $(A \rightarrow \neg \neg A, \neg \neg A \rightarrow A)$ from the system $\mathrm{B}$. The basic logic $\mathrm{B}$ is also especially significant among relevant logics because is used as a starting system to define a wealth of extensions interpretable with the R-M semantics (cf. [11, chapter 4]).

Robles and Méndez developed the logic E4 [10], another interesting 4 -valued logic which was built upon a modification of the conditional function of MBN4 (cf. Definition 2.1) and is also a proper extension of Routley and Meyer's logic B. Robles and Méndez suggested that E4 could be seen as the "4-valued logic of (relevant) entailment" whereas BN4 could be considered as the "4-valued logic of the relevant conditional". The main reason for this is that they believe that E4 is related to BN4 in a similar way to 
which Anderson and Belnap's logic of entailment $\mathrm{E}$ is related to the system R (cf. [1] about the logics E and R). In particular, according to Méndez and Robles, BN4 can intuitively be described as a 4-valued extension of contractionless relevant logic $\mathrm{R}(\mathrm{RW})$ and $\mathrm{E} 4$ as a 4-valued extension of reductioless logic $\mathrm{Er}^{1}$.

Although E4 was presented as a companion to BN4 worthy of consideration, Robles and Méndez asserted that E4 might not be the only alternative to $\mathrm{BN} 4$ and set out six different variations of the conditional function of MBN4 which could turn out to be possibly interesting 4-valued logics in the family of relevant logics. Some research on the logics built upon these tables has recently been conducted in [6]. In particular, it has been proved that they are the only variants of MBN4 and ME4 which verify Routley and Meyer's logic B. Furthermore, they have been endowed with a BelnapDunn semantics. The aim of this paper is to provide a general reduced Routley-Meyer type semantics for those logics in order to connect them to the wide range of logics interpretable with this semantics and specially to the systems BN4 and E4, which have been also already interpreted by the R-M type semantics [9]. It is worth underlining that: (I) validity of formulae depends on a singleton in the reduced general Routley-Meyer semantics (i.e., the set of designated points is limited to a single element); (II) reduced models are generally preferable when there is the possibility to define them $($ cf. $[4,5])$. As a matter of fact, we face some problems when defining reduced models for the majority of the logics here considered given the apparent ineliminability of disjunctive rules. However, these inconvenients are solved according to the methodology suggested in $([4,5,11])$.

The structure of this paper proceeds as follows. In Section 2, the implicative variants of MBN4 and ME4 which verify Routley and Meyer's logic $\mathrm{B}$ are displayed. In Section 3, a basic logic which serves a mere instrumental role - the logic b4 - is presented and also extended to each of the logics considered in this paper. Next, reduced general Routley-Meyer semantics for the logic b4 is provided in Section 4. In Section 5 and Section 6, a series of lemmas and notions for extensions of the logic b4-Eb4-logics - are recalled as they were already proved in previous papers $([3,9])$ following the method described in [11, chapter 4]. Finally, completeness theorems for

${ }^{1} \mathrm{RW}$ will be the result of dropping the axiom contraction $([A \rightarrow(A \rightarrow B)] \rightarrow(A \rightarrow$ $B))$ from $\mathrm{R}$ and Er the result of dropping the axiom reductio $((A \rightarrow \neg A) \rightarrow \neg A)$ from E. The label Er is Robles and Méndez's. 
b4 and its extensions are proved in Section 7 and Section 8, respectively. In particular, in Section 8, the essential postulates of the extensions of b4 considered in this paper are displayed and proved adequate for Eb4-models where their corresponding axiom is valid.

\section{Implicative variants of MBN4 and ME4 which verify Routley and Meyer's logic B}

In this section, I display the matrices upon which the logics considered in this paper were built, i.e., the implicative variants of MBN4 and ME4 which verify Routley and Meyer's logic B (cf. [11, chapter 4]).

First, the notions of Languages and Logics are fairly standard (cf. [9]). The propositional language $\mathcal{L}$ consists of a denumerable set of propositional variables $p_{0}, p_{1} \ldots, p_{n}, \ldots$ and some or all of the following connectives $\rightarrow, \wedge$, $\vee, \neg . A, B, C$, etc. are metalinguistic variables. Logics are formulated as Hilbert-style axiomatic systems. The notions of proof and theorem are understood as it is customary $\left(\Gamma \vdash_{L} A\right.$ means that $A$ is derivable from the set of wffs $\Gamma$ in the logic $L$; and $\vdash_{L} A$ means that $A$ is a theorem of the logic $L)$.

Next, I introduce the matrices upon which systems BN4 and E4 are built.

Definition 2.1 (Brady's matrix MBN4 and Robles and Méndez's matrix ME4). The propositional language $\mathcal{L}$ consists of the connectives $\rightarrow, \wedge, \vee$ and $\neg$. Brady's matrix MBN4 and Robles and Méndez's matrix ME4 are the structures $\langle\mathcal{V}, \mathcal{D}, \digamma\rangle$, where (i) $\mathcal{V}$ is $\{0,1,2,3\}$ and it is partially ordered as shown in the following lattice:

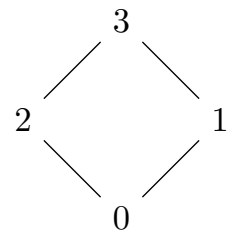

(ii) $\mathcal{D}=\{2,3\}$; (iii) $\digamma=\left\{f_{\rightarrow}, f_{\wedge}, f_{\vee}, f_{\neg}\right\}$ where $f_{\wedge}$ and $f_{\vee}$ are defined as the glb (or lattice meet) and the lub (or lattice join), respectively. $f_{\neg}$ is an 
involution with $f_{\neg}(0)=3, f_{\neg}(3)=0, f_{\neg}(1)=1$ and $f_{\neg}(2)=2$. Tables for $\wedge, \vee$ and $\neg$ are now displayed.

\begin{tabular}{|c|c|c|c|c|c|c|c|c|c|c|c|c|c|c|}
\hline$\wedge$ & 0 & 1 & 2 & 3 & V & 0 & 1 & 2 & 3 & & & & & \\
\hline 0 & 0 & 0 & 0 & 0 & 0 & 0 & 1 & 2 & 3 & & & & & \\
\hline 1 & 0 & 1 & 0 & 1 & 1 & 1 & 1 & 3 & 3 & 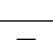 & 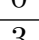 & & $\frac{2}{2}$ & 0 \\
\hline 2 & 0 & 0 & 2 & 2 & 2 & 2 & 3 & 2 & 3 & & & & 2 & 0 \\
\hline 3 & 0 & 1 & 2 & 3 & 3 & 3 & 3 & 3 & 3 & & & & & \\
\hline
\end{tabular}

Finally, $f_{\rightarrow}$ is defined in each system according to the following tables ${ }^{2}$ :

\begin{tabular}{|c|c|c|c|c|c|c|c|c|c|c|c|}
\hline \multirow{5}{*}{ t1 (BN4) } & $\rightarrow$ & 0 & 1 & 2 & 3 & \multirow{5}{*}{ t5 (E4) } & $\rightarrow$ & 0 & 1 & 2 & 3 \\
\hline & 0 & 3 & 3 & 3 & 3 & & 0 & 3 & 3 & 3 & 3 \\
\hline & 1 & 1 & 3 & 1 & 3 & & 1 & 0 & 2 & 0 & 3 \\
\hline & 2 & 0 & 1 & 2 & 3 & & 2 & 0 & 0 & 2 & 3 \\
\hline & 3 & 0 & 1 & 0 & 3 & & 3 & 0 & 0 & 0 & 3 \\
\hline
\end{tabular}

Definition 2.2 (Variants of MBN4 and ME4 considered in this paper). Each Mt $i(1 \leq i \leq 8)$ is a structure $(\mathcal{V}, \mathcal{D}, \digamma)$ where $\mathcal{V}, \mathcal{D}, f_{\wedge}, f_{\vee}$ and $f_{\neg}$ are defined as in MBN4 and ME4 (cf. Definition 2.1) and $f \rightarrow$ is defined according to the corresponding $\mathrm{t} i$ below ( $\mathrm{t} 1$ and $\mathrm{t} 5$ are left out here to refer to $\mathrm{BN} 4$ and $\mathrm{E} 4$, respectively):

\begin{tabular}{|c|c|c|c|c|c|c|c|c|c|c|c|c|c|c|c|c|c|}
\hline & $\rightarrow$ & 0 & 1 & 2 & 3 & & $\rightarrow$ & 0 & 1 & 2 & 3 & & $\rightarrow$ & 0 & 1 & 2 & 3 \\
\hline & 0 & 3 & 3 & 3 & 3 & & 0 & 3 & 3 & 3 & 3 & & 0 & 3 & 3 & 3 & 3 \\
\hline \multirow[t]{5}{*}{$\mathrm{t} 2$} & 1 & 0 & 3 & 0 & 3 & t3 & 1 & 1 & 3 & 1 & 3 & $\mathrm{t} 4$ & 1 & 0 & 3 & 0 & 3 \\
\hline & 2 & 0 & 0 & 2 & 3 & & 2 & 0 & 0 & 2 & 3 & & 2 & 0 & 1 & 2 & 3 \\
\hline & 3 & 0 & 0 & 0 & 3 & & 3 & 0 & 0 & 0 & 3 & & 3 & 0 & 1 & 0 & 3 \\
\hline & $\rightarrow$ & 0 & 1 & 2 & 3 & & $\rightarrow$ & 0 & 1 & 2 & 3 & & $\rightarrow$ & 0 & 1 & 2 & 3 \\
\hline & 0 & 3 & 3 & 3 & 3 & & 0 & 3 & 3 & 3 & 3 & & 0 & 3 & 3 & 3 & 3 \\
\hline \multirow[t]{3}{*}{ t6 } & 1 & 0 & 2 & 0 & 3 & $\mathrm{t} 7$ & 1 & 0 & 2 & 1 & 3 & t8 & 1 & 0 & 2 & 1 & 3 \\
\hline & 2 & 0 & 1 & 2 & 3 & & 2 & 0 & 0 & 2 & 3 & & 2 & 0 & 1 & 2 & 3 \\
\hline & 3 & 0 & 0 & 0 & 3 & & 3 & 0 & 0 & 0 & 3 & & 3 & 0 & 0 & 0 & 3 \\
\hline
\end{tabular}

Remark 2.3 (Implicative variants of MBN4 and ME4 which verify Routley and Meyer's logic B). The matrices considered in this paper are the only

${ }^{2}$ From now on, the labels t 1 and t5 will be used to refer to the implicative tables of BN4 and E4, respectively, since labels t2-t4 will be used to refer to the implicative tables of the variants of BN4 and, likewise, t6-t8 will be used for those of the implicative variants of $\mathrm{E} 4$. 
implicative variants of MBN4 (t2-t4) and ME4 (t6-t8) which verify Routley and Meyer's logic B (cf. [11, Chapter 4]). This was already proved as a Proposition in [6, Proposition 3.2].

\section{The basic logic b4 and its extensions}

The eight logics considered in this paper are developed in this section as implicative extensions of b4. Therefore, the basic logic b4 is a system contained in every Lt $i$-logic $(1 \leq i \leq 8)$, i.e., in every logic built upon the matrices characterized by the implicative tables displayed in Section 2 (cf. Definitions 2.1 and 2.2). As a matter of fact, the label b4 is intended to abbreviate "basic logic contained in every companion of BN4 or E4 which includes Routley and Meyer's logic B". In the following sections, b4 will be used as a common ground for the soundness and completeness proofs.

DeFinition 3.1 (The basic logic b4). The logic b4 is axiomatized with the following axioms and rules ADJ, MP, dMP, dPREF, dSUF, dCON, dCTE displayed below:

Axioms

$$
\begin{aligned}
& \text { A1 } A \rightarrow A \\
& \text { A2 }(A \wedge B) \rightarrow A /(A \wedge B) \rightarrow B \\
& \text { A3 }[(A \rightarrow B) \wedge(A \rightarrow C)] \rightarrow[A \rightarrow(B \wedge C)] \\
& \text { A4 } A \rightarrow(A \vee B) / B \rightarrow(A \vee B) \\
& \text { A5 }[(A \rightarrow C) \wedge(B \rightarrow C)] \rightarrow[(A \vee B) \rightarrow C] \\
& \text { A6 }[A \wedge(B \vee C)] \rightarrow[(A \wedge B) \vee(A \wedge C)] \\
& \text { A7 } \neg \neg A \rightarrow A \\
& \text { A8 } A \rightarrow \neg \neg A \\
& \text { A9 } \neg A \rightarrow[A \vee(A \rightarrow B)] \\
& \mathrm{A} 10 B \rightarrow[\neg B \vee(A \rightarrow B)] \\
& \mathrm{A} 11(A \vee \neg B) \vee(A \rightarrow B) \\
& \mathrm{A} 12(A \rightarrow B) \vee[(\neg A \wedge B) \rightarrow(A \rightarrow B)] \\
& \mathrm{A} 13 A \rightarrow[B \rightarrow[[(A \vee B) \vee \neg(A \vee B)] \vee(A \rightarrow B)]]
\end{aligned}
$$


Rules of inference

Adjunction: $A, B \Rightarrow A \wedge B$

Modus Ponens: $A, A \rightarrow B \Rightarrow B$

Disjunctive Modus Ponens: $C \vee A, C \vee(A \rightarrow B) \Rightarrow C \vee B$

Disjunctive prefixing: $C \vee(A \rightarrow B) \Rightarrow C \vee[(D \rightarrow A) \rightarrow(D \rightarrow B)]$

Disjunctive suffixing: $C \vee(A \rightarrow B) \Rightarrow C \vee[(B \rightarrow D) \rightarrow(A \rightarrow D)]$

Disjunctive Contraposition: $C \vee(A \rightarrow B) \Rightarrow C \vee(\neg B \rightarrow \neg A)$

Disjunctive Counterexample: $C \vee(A \wedge \neg B) \Rightarrow C \vee \neg(A \rightarrow B)$

Remark 3.2 (About b4). b4 is the result of adding the axioms A9-A13 and rules dMP, dPREF, dSUF, dCON and dCTE to Routley and Meyer's basic logic B (cf. [11, Chapter 4]). As a matter of fact, b4 can be seen as an extension of $\mathrm{dB}$ (i.e., the disjunctive version of Routley and Meyer's $\operatorname{logic} \mathrm{B})$.

Next, I prove some theorems of b4 which will be useful throughout this paper.

Proposition 3.3 (Some theorems and rules of b4). The following theorems and rules are derivable in $\mathrm{b} 4$.

T1 $A \leftrightarrow(A \vee A)$

T2 $[(A \rightarrow B) \wedge(C \rightarrow D)] \rightarrow[(A \wedge C) \rightarrow(B \wedge D)]$

T3 $[A \vee(B \vee C)] \leftrightarrow[(A \vee B) \vee C]$

T4 $(A \rightarrow B) \rightarrow[A \rightarrow(B \vee C)]$

T5 $(A \rightarrow B) \rightarrow[(A \wedge C) \rightarrow(B \vee D)]$

T6 $\neg(A \wedge B) \leftrightarrow(\neg A \vee \neg B)$

T7 $\quad(\neg A \wedge \neg B) \leftrightarrow \neg(A \vee B)$

T8 $A \rightarrow[\neg A \vee(\neg A \rightarrow B)]$

T9 $\neg A \rightarrow[B \vee[(A \wedge B) \rightarrow C]]$

TRAN $A \rightarrow B, B \rightarrow C \Rightarrow A \rightarrow C$

$\mathrm{SUM} \quad A \rightarrow B \Rightarrow(A \vee C) \rightarrow(B \vee C)$ 
Every correspondent non-disjunctive version of the rules of b4 except for the rule MP (i.e., PREF, SUF, CON, CTE) is also a derived rule of b4.

PRoOF: T1-T7, SUM and TRAN are theorems and rules of the system B. T8 is obtained by A8, A9 and TRAN. T9 can be proved using A2, A9 and rules TRAN, CON and SUM. Finally, rules PREF, SUF, CON and CTE can easily be derived from their disjunctive version plus the rule MP, A4 and T1.

In the following lines, I introduce the extensions of b4 which I have referred to from the beginning of the section. In the first place, I define the notion of extensions (and expansions) of a propositional logic.

Definition 3.4 (Extensions and expansions of a propositional logic L). Let $L$ be a logic formulated with axioms $a_{1}, \ldots, a_{n}$ and rules of derivation $r_{1}, \ldots, r_{m}$. A logic $L^{\prime}$ includes $L$ iff $a_{1}, \ldots, a_{n}$ are theorems of $L^{\prime}$ and rules $r_{1}, \ldots, r_{m}$ are provable in $L^{\prime}$. If such were the case, $L^{\prime}$ would be either an extension of $L$ (i.e., a strengthening of $L$ in the language of $L$ ) or an expansion of it (i.e., a strengthening of $L$ in an expansion of the language of $L$ ). We shall generally refer to extensions of a logic $L$ by $E L$-logics.

Definition 3.5 (Extensions of b4 considered in this paper-Lti-logics). We refer by Lti $(1 \leq i \leq 8)$ to the eight extensions of b4 considered in this paper, these are, BN4 (Lt1), E4 (Lt5) and the logics characterized by the implicative variants of MBN4 and ME4 (Lt2-Lt4 and Lt6-Lt8, respectively). Each Lt $i$-logic is the result of adding the following axioms (from the list below) to b4:

Lt1 (BN4): A14-A16

Lt2: A17-A23

Lt3: A14, A15, A18, A19, A22-A24

Lt4: A16, A17, A20-A22

Lt5 (E4): A17-A21, A23, A25-A27

Lt6: A17, A20, A21, A23, A26, A28, A29

Lt7: A14, A18, A19, A21, A23, A26, A30

Lt8: A14, A21, A23, A26, A29, A30 
Now, I display the list of axioms from which the Lt $i$-logics are built:

$\mathrm{A} 14(A \wedge \neg B) \rightarrow[(A \wedge \neg B) \rightarrow \neg(A \rightarrow B)]$

$\mathrm{A} 15 \quad A \vee[\neg(A \rightarrow B) \rightarrow A]$

$\mathrm{A} 16 \neg B \vee[\neg(A \rightarrow B) \rightarrow \neg B]$

$\mathrm{A} 17[A \wedge(A \rightarrow B)] \rightarrow B$

$\mathrm{A} 18[(A \rightarrow B) \wedge \neg B] \rightarrow \neg A$

$\mathrm{A} 19 \quad A \rightarrow[B \vee \neg(A \rightarrow B)]$

$\mathrm{A} 20 \neg B \rightarrow[\neg A \vee \neg(A \rightarrow B)]$

$\mathrm{A} 21[\neg(A \rightarrow B) \wedge \neg A] \rightarrow A$

$\mathrm{A} 22 \neg(A \rightarrow B) \rightarrow(A \vee \neg B)$

$\mathrm{A} 23[\neg(A \rightarrow B) \wedge B] \rightarrow \neg B$

$\mathrm{A} 24 B \rightarrow\{[B \wedge \neg(A \rightarrow B)] \rightarrow A]\}$

$\mathrm{A} 25(A \rightarrow B) \vee \neg(A \rightarrow B)$

A26 $(\neg A \vee B) \vee \neg(A \rightarrow B)$

$\mathrm{A} 27[(A \rightarrow B) \wedge(A \wedge \neg B)] \rightarrow \neg(A \rightarrow B)$

$\mathrm{A} 28 \neg(A \rightarrow B) \vee[(A \wedge \neg B) \rightarrow \neg(A \rightarrow B)]$

A29 $\{[\neg(A \rightarrow B) \wedge \neg A] \rightarrow \neg B\} \vee \neg B$

$\mathrm{A} 30\{[\neg(A \rightarrow B) \wedge B] \rightarrow A\} \vee A$ 


\section{Eb4-models in Routley-Meyer ternary relational semantics}

In this section, the soundness of b4 is proved, but I establish the setting within which an RM-semantics for any Eb4-logic can be developed. In this sense, some of the facts upon which the soundness proof leans upon are applicable to Eb4-logics in general. First, we underline that the label EL will be used to refer to an extension of the logic L (cf. Definition 3.4). Now, we set the notion of Eb4-models.

Definition 4.1 (Eb4-models). An Eb4-model $\mathrm{M}$ is a structure $<T, K$, $R, *, \vDash>$ where $K$ is a non-empty set, $T \in K, R$ is a ternary relation on $K$ and $*$ is a unary operator on $K$ subject (at least) to the following definitions and postulates for all $a, b, c \in K$ :

$\mathrm{d} 1 \quad a \leq b={ }_{d f} R T a b$

$\mathrm{d} 2 a=b={ }_{d f} a \leq b \& b \leq a$

$\mathrm{d} 3 R^{2} a b c d={ }_{d f}(\exists x \in K)(R a b x \& R x c d)$

p1 $a \leq a$

p2 $(a \leq b \& R b c d) \Rightarrow R a c d$

p3 $R^{2} T a b c \Rightarrow(\exists x \in K)(R T b x \& \operatorname{Raxc})$

$\mathrm{p} 4 R^{2} T a b c \Rightarrow(\exists x \in K)(R a b x \& R T x c)$

p5 $a^{* *} \leq a$

p6 $a \leq a^{* *}$

p7 $a \leq b \Rightarrow b^{*} \leq a^{*}$

p8 $R T^{*} T T^{*}$

p9 $R a b c \Rightarrow\left(b \leq a^{*}\right.$ or $\left.b \leq a\right)$

p10 Rabc $\Rightarrow\left(a \leq c\right.$ or $\left.a^{*} \leq c\right)$

p11 $R T a b \Rightarrow\left(T^{*} \leq b\right.$ or $\left.a \leq T\right)$

$\mathrm{p} 12\left(R T a b \& R^{2} T c d e\right) \Rightarrow\left(a \leq c^{*}\right.$ or $d \leq c^{*}$ or $c \leq b$ or $\left.c \leq e\right)$

$\mathrm{p} 13(R a b c \& R c d e) \Rightarrow\left(a \leq c\right.$ or $b \leq c$ or $c^{*} \leq c$ or $d \leq c$ or $\left.b \leq e\right)$ 
Finally, $\vDash$ is a valuation relation from $K$ to the set of all wffs such that the following conditions (clauses) are satisfied for every propositional variable $p$, wffs $A, B$ and $a \in K$ :

(i) $(a \leq b \& a \vDash p) \Rightarrow b \vDash p$

(ii) $a \vDash A \wedge B$ iff $a \vDash A \& a \vDash B$

(iii) $a \vDash A \vee B$ iff $a \vDash A$ or $a \vDash B$

(iv) $a \vDash A \rightarrow B$ iff for all $b, c \in K,(R a b c \& b \vDash A) \Rightarrow c \vDash B$

(v) $a \vDash \neg A$ iff $a^{*} \not \models A$

A structure $<T, K, R, *, \vDash>$ like the latest represent the most basic Eb4-model. On the other hand, other different Eb4-models can be defined adding new postulates to those of the basic Eb4-model presented above (i.e., p1-p13). As a matter of fact, reduced semantics for the Lti-logics displayed in Definition 3.5 (which are indeed Eb4-logics) are defined in Section 8 following the aforementioned method.

Definition 4.2 (b4-models). A b4-model is a Eb4-model with no additional postulate.

In what follows, the definitions of truth, validity and semantic consequence in a class of Eb4-models are defined.

Definition 4.3 (Truth in a class of Eb4-models). Let $\mathfrak{M}$ be a class of Eb4-models and $\mathrm{M} \in \mathfrak{M}$. A wff $A$ is true in $\mathrm{M}$ iff $T \vDash A$ in this model.

Definition 4.4 (Validity in a class of Eb4-models). Let $\mathfrak{M}$ be a class of Eb4-models. A wff $A$ is valid in $\mathfrak{M}$ (in symbols, $\vDash A$ ) iff $T \vDash A$ in all $\mathrm{M} \in \mathfrak{M}$.

Definition 4.5 (Semantic consequence in a class of Eb4-models). Let $\mathfrak{M}$ be a class of Eb4-models. Then, for all $\mathrm{M} \in \mathfrak{M}$ and any set of wffs $\Gamma$ and wff $A: \Gamma \vDash_{\mathrm{M}} A$ ( $A$ is a semantic consequence of $\Gamma$ in the model $\left.\mathrm{M}\right)$ iff $T \vDash A$ if $T \vDash \Gamma(T \vDash \Gamma$ iff $T \vDash B$ for all $B \in \Gamma)$. Then, $\Gamma \vDash_{\mathfrak{M}} A$ ( $A$ is a semantic $\mathfrak{M}$-consequence of $\Gamma$ ) iff $\Gamma \vDash_{\mathrm{M}} A$ for all $\mathrm{M} \in \mathfrak{M}$.

Next, we specify what (in our view) constitutes a semantics and when a logic is endowed with a semantics. 
Definition 4.6 (Reduced general Routley-Meyer semantics for Eb4-logics). Let L be a Eb4-logic. $\Sigma=\{\mathfrak{M}, \models\}$ is a semantics for L iff L is sound and complete with respect to $\Sigma$. If this condition is fulfilled, we establish that L-models (i.e., $M \in \mathfrak{M}$ ) together with the definition of validity in $\mathfrak{M}$ constitute a Routley-Meyer semantics for L.

The following couple of lemmas is needed to prove that the system b4 is sound w.r.t. the semantics just defined.

Lemma 4.7 (Hereditary condition). Let $\mathfrak{M}$ be a class of Eb4-models. For any $\mathrm{M} \in \mathfrak{M}, a, b \in K$ and wff $A:(a \leq b \& a \vDash A) \Rightarrow b \vDash A$.

Proof: By induction on the length of $A$. The conditional case is proved with $\mathrm{p} 2$ and the negation case with $\mathrm{p} 7$ and $\mathrm{d} 1$.

Lemma 4.8 (Entailment Lemma). Let $\mathfrak{M}$ be a class of Eb4-models. For any wffs $A, B$, we have $\vDash_{\mathfrak{M}} A \rightarrow B$ iff $a \vDash A \Rightarrow a \vDash B$ for all $a \in K$ in all $\mathrm{M} \in \mathfrak{M}$.

Proof: $(\Rightarrow)$ By p1 and d1. $(\Leftarrow)$ By d1 and Lemma 4.7.

Proposition $4.9\left(\Gamma \vdash_{b 4} A \Rightarrow \Gamma \vDash_{\mathrm{M}} A\right)$. Let $\mathfrak{M}$ be a class of Eb4-models. For any set of wffs $\Gamma$, wff $A$ and $\mathrm{M} \in \mathfrak{M}$, if $\Gamma \vdash_{b 4} A$, then $\Gamma \vDash_{\mathrm{M}} A$.

Proof: We have to prove three different cases: (i) $A \in \Gamma$; (ii) $A$ is an axiom; (iii) $A$ is derived by means of any rule of $\mathrm{b} 4$. In the first place, case (i) is trivial. As for the case (ii), it will be proved that the axioms are valid in any class of Eb4-model. The validity of axioms A1-A8 is proved as in [11, Chapter 4] and that of A9 and A11 as (the validity of A10 and A13, respectively) in [9, p. 12]. Now, A10, A12 and A13 will be proved. We lean upon the Entailment Lemma (Lemma 4.8) and proceed by reductio ad absurdum. We also use the Hereditary Condition (Lemma 4.7) and clauses (ii)-(v) in Definition 4.1. Furthermore, the postulates displayed in Definition 4.1 will be used to prove the validity of the named axioms.

(A10) $B \rightarrow[\neg B \vee(A \rightarrow B)]$ is valid in any Eb4-model. Suppose that there are $a \in K$ in some Eb4-model $\mathrm{M}$ and wffs $A, B$ such that (1) $a \vDash B$ but (2) $a \not \models \neg B \vee(A \rightarrow B)$. By clause (iii) of Definition 4.1, (3) $a \not \models \neg B$ $\&$ (4) $a \not \models A \rightarrow B$. By clause (v) of the same definition and 3, (5) $a^{*} \vDash B$. Then, there are $b, c \in K$ such that (6) Rabc, (7) $b \vDash A$, (8) $c \not \models B$ by 4 and clause (iv). Now, given 6 and p10 $\left(R a b c \Rightarrow\left(a \leq c\right.\right.$ or $\left.\left.a^{*} \leq c\right)\right)$, we have 
$a \leq c$ or $a^{*} \leq c$. Finally, (9) $c \vDash B$ by applying the Hereditary Condition (Lemma 4.7) to either 1 or 5 . However, 8 contradicts 9 .

(A12) $(A \rightarrow B) \vee[(\neg A \wedge B) \rightarrow(A \rightarrow B)]$ is valid in any Eb4-model. Suppose that there is some Eb4-model $\mathrm{M}$ and wffs $A, B$ such that (1) $T \not \models(A \rightarrow B) \vee[(\neg A \wedge B) \rightarrow(A \rightarrow B)]$. Then, (2) $T \not \nvdash A \rightarrow B$ and (3) $T \not \models(\neg A \wedge B) \rightarrow(A \rightarrow B)$. By clause (iv), (4) RTab, (5) $a \vDash A$, (6) $b \not \models B$, for some $a, b \in K$ - given 2-and (7) RTcx, (8) $c \vDash \neg A \wedge B$ and (9) $x \not \models A \rightarrow B$ for some $c, x \in K$ - given 3. Again, by applying clause (iv) to 9 , we get (10) Rxde, (11) $d \vDash A$ and (12) $e \not \models B$, for some $d, e \in K$. Similarly, by applying clause (ii) to 8 , we obtain (13) $c \vDash \neg A$ (i.e., $c^{*} \not \models A$ ) and $c \vDash B$. Now, given p12 ( RTab\& $\left.R^{2} T c d e\right) \Rightarrow\left(a \leq c^{*}\right.$ or $d \leq c^{*}$ or $c \leq b$ or $c \leq e)), 4,7$ and 10, we have $a \leq c^{*}$ or $d \leq c^{*}$ or $c \leq b$ or $c \leq e$. Let us suppose $a \leq c^{*}$ or $d \leq c^{*}$, then we have (14) $c^{*} \vDash A$-contradicting 13-by applying the Hereditary Condition to either 5 or 11, respectively. Next, let us suppose $c \leq b$, then we get (15) $b \vDash B$ similarly, given 13 . However, 15 contradicts 6 . Finally, let us suppose $c \leq e$, we get (16) $e \vDash B$ (given 13), which contradicts 12 .

(A13) $A \rightarrow[B \rightarrow[[(A \vee B) \vee \neg(A \vee B)] \vee(A \rightarrow B)]]$ is valid in any Eb4-model. Suppose that there is some Eb4-model $\mathrm{M}$ and wffs $A, B$ such that (1) $a \vDash A$ but $(2) a \not \models B \rightarrow[[(A \vee B) \vee \neg(A \vee B)] \vee(A \rightarrow B)]$. By clause (iv), (3) Rabc, (4) $b \vDash B$ and (5) $c \not \models[(A \vee B) \vee \neg(A \vee B)] \vee(A \rightarrow B)$, for $b, c \in K$. Then, we get (6) $c \not \models A \vee B$ (i.e., $c \not \models A \& c \not \models B$ ), (7) $c \not \models \neg(A \vee B)$ (i.e., $c^{*} \vDash A \vee B$ ), (8) $c \not \models A \rightarrow B$, by clause (iii). Now, by clause (iv), we have (9) $d \vDash A$ and (10) $e \not \models B$ for some $d, e \in K$ such that (11) Rcde. Then, $a \leq c$ or $b \leq c$ or $c^{*} \leq c$ or $d \leq c$ or $b \leq e$ given 3,11 and $\mathrm{p} 13\left((\right.$ Rabc \& Rcde $) \Rightarrow\left(a \leq c\right.$ or $b \leq c$ or $c^{*} \leq c$ or $d \leq c$ or $\left.\left.b \leq e\right)\right)$. Similarly as the proof of A12, we can easily see that a contradiction is reached whatever the case may be given the Hereditary Condition plus 1 , 4, 6, 7, 9 and 10 .

Case (iii), $A$ is the result of applying a rule of $\mathrm{b} 4$, has on its own several subcases. The subcase of ADJ is trivial and the subcases when $A$ is derived by MP and dMP are proved as in [9, Theorems 3.7, 3.10]. The remaining disjunctive rules are proved similarly ${ }^{3}$. As an example, let us prove the subcase when $A$ is the result of applying the rule dCTE. Suppose $\Gamma \vDash_{\mathrm{M}} D \vee(B \wedge \neg C)$ for some wffs $B, C$ and $D$. Furthermore, suppose $T \vDash \Gamma$.

${ }^{3}$ The reader can also find the proofs for some of these rules displayed in [11, Chapter 4, pp. 336, ff.]. 
Then, (1) $T \vDash D \vee(B \wedge \neg C)$ and by reductio, (2) $T \not \models D \vee \neg(B \rightarrow C)$. Now, we have (3) $T \vDash D$ or $T \vDash B \wedge \neg C$ and (4) $T \not \models D \& T \not \models \neg(B \rightarrow C)$ (i.e., $T^{*} \vDash B \rightarrow C$ ) by applying clause (iii) to 1 and 2 , respectively. Then, obviously, (5) $T \vDash B \wedge \neg C$ (i.e., $T \vDash B \& T^{*} \not \models C$ ). Now, by applying clause (iv) to $\mathrm{p} 8\left(R T^{*} T T^{*}\right), 4$ and 5 , we obtain $(6) T^{*} \vDash C$. However, 5 contradicts 6 .

TheOrem 4.10 (Soundness of b4). For any set of wffs $\Gamma$ and wff $A$ : If $\Gamma \vdash_{b 4} A$, then $\Gamma \vDash_{b 4} A$.

Proof: It is trivial given Proposition 4.9.

\section{Extension and primeness lemmas}

In the present section, we shall introduce the extension lemmas. Firstly, we set the notion of Eb4-theories and several other related notions. We also display a couple of definitions and some lemmas which will be crucial points in the completeness theorem proved in Section 7. In these lemmas, we apply the method developed in "Relevant logics and their rivals I" (cf. [11, Chapter 4]) and followed by Brady (cf. [3, pp. 24-25]). We shall omit some of those proofs since they are similar to Brady's ${ }^{4}$ [3].

In the first place, we set some preliminary definitions.

Definition 5.1 (Eb4-theories). Let L be an Eb4-logic. An L-theory $\mathcal{T}$ is a set of wffs closed under Adjunction (Adj) and provable L-entailment (L-ent). That is to say, a set of wffs is closed under Adj iff, whenever $A$, $B \in \mathcal{T}$, then $A \wedge B \in \mathcal{T}$; a set of wffs is closed under L-ent iff, whenever $A \rightarrow B$ is a theorem of $\mathrm{L}$ and $A \in \mathcal{T}$, then $B \in \mathcal{T}$.

Definition 5.2 (Types of Eb4-theories). Let L be an Eb4-logic and $\mathcal{T}$ an L-theory. We set (1) $\mathcal{T}$ is prime iff, for wffs $A$ and $B$, whenever $A \vee B \in \mathcal{T}$, then either $A \in \mathcal{T}$ or $B \in \mathcal{T}$; (2) $\mathcal{T}$ is regular iff $\mathcal{T}$ contains all theorems in $\mathrm{L} ;(3) \mathcal{T}$ is trivial iff it contains every wff; (4) $\mathcal{T}$ is a-consistent (consistent in an absolute sense) iff $\mathcal{T}$ is not trivial; (5) $\mathcal{T}$ is empty iff it contains no wff.

\footnotetext{
${ }^{4}$ We shall also follow Robles and Méndez's structure and method for the extension lemmas (cf. [10, pp. 845-847]). In addition, some of the outlined proofs were already given in $[6,9]$.
} 
Definition 5.3 (Sets of wffs closed under a certain rule). For any wffs $A$, $B, C$ and $D$, a set of wffs $\Gamma$ is closed by: (1) $M P$ iff $A \rightarrow B \in \Gamma$ and $A \in \Gamma$, then $B \in \Gamma$; (2) $d M P$ iff $(A \rightarrow B) \vee C \in \Gamma$ and $A \vee C \in \Gamma$ then $B \vee C \in \Gamma$; (3) $C O N$ iff $A \rightarrow B \in \Gamma$, then $\neg B \rightarrow \neg A \in \Gamma$; (4) dCON iff $(A \rightarrow B) \vee C \in \Gamma$, then $(\neg B \rightarrow \neg A) \vee C \in \Gamma$; (5) PREF iff $A \rightarrow B \in \Gamma$, then $(C \rightarrow A) \rightarrow(C \rightarrow B) \in \Gamma$; (6) dPREF iff $(A \rightarrow B) \vee D \in \Gamma$, then $[(C \rightarrow A) \rightarrow(C \rightarrow B)] \vee D \in \Gamma ;$ (7) SUF iff $A \rightarrow B \in \Gamma$, then $(B \rightarrow C) \rightarrow(A \rightarrow C) \in \Gamma$; (8) dSUF iff $(A \rightarrow B) \vee D \in \Gamma$, then $[(B \rightarrow C) \rightarrow(A \rightarrow C)] \vee D \in \Gamma$; (9) CTE iff $A \wedge \neg B \in \Gamma$, then $\neg(A \rightarrow B) \in \Gamma$; (10) dCTE iff $(A \wedge \neg B) \vee C \in \Gamma$, then $\neg(A \rightarrow B) \vee C \in \Gamma$; (11) $M T$ iff $A \rightarrow B \in \Gamma$ and $\neg B \in \Gamma$, then $\neg A \in \Gamma$; (12) TRAN iff $A \rightarrow B \in \Gamma$ and $B \rightarrow C \in \Gamma$, then $A \rightarrow C \in \Gamma$.

Definition 5.4 (Full regularity). Let L be an Eb4-logic, an L-theory $\mathcal{T}$ is fully regular iff it is a regular L-theory (cf. Definitions 5.1 and 5.2) which is closed under the rules of b4 (i.e., MP, dMP, dCON, dPREF, dSUF, dCTE; cf. Definition 5.3).

Proposition 5.5 (Derived rules under which fully regular Eb4-theories are closed). Let $\mathrm{L}$ be an Eb4-logic, if $\mathcal{T}$ is a fully regular L-theory, then it is closed under (1) CON, (2) PREF, (3) SUF, (4) CTE, (5) MT and (6) TRAN.

Proof: Cases (1)-(4): by A4 and T1 $(A \leftrightarrow(A \vee A))$ and the fact that $\mathcal{T}$ is fully regular (i.e., closed under dCON, dPREF, dSUF and dCTE, respectively for each case). Cases (5)-(6): by hypothesis, $\mathcal{T}$ is fully regular (therefore closed under MP) and by the fact that $\mathcal{T}$ is closed under CON and SUF (given what has already been proved in cases (1) and (3)), respectively for each case.

Definition 5.6 (Disjunctive Eb4-derivability). Let L be an Eb4-logic, $\Gamma$ and $\Theta$ be non-empty sets of wffs, $\Theta$ is disjunctively derivable from $\Gamma$ in Eb4 (in symbols, $\Gamma \vdash_{L}^{d} \Theta$ ) iff $A_{1} \wedge \ldots \wedge A_{n} \vdash_{L} B_{1} \vee \ldots \vee B_{n}$ for some wffs $A_{1}, \ldots, A_{n} \in \Gamma$ and $B_{1}, \ldots, B_{n} \in \Theta$.

The following lemma is essential in order to prove the Extension to maximal sets lemma (Lemma 5.9). 
Lemma 5.7 (Preliminary lemma to the extension lemma). Let $L$ be an Eb4-logic closed under no other rules than those specified in Definition 5.4. For any wffs $A, B_{1}, \ldots, B_{n}$, if $\left\{B_{1}, \ldots, B_{n}\right\} \vdash_{L} A$, then, for any wff $C$, $C \vee\left(B_{1} \wedge \ldots \wedge B_{n}\right) \vdash_{L} C \vee A$.

Proof: Induction on the length of $A$ (cf. p. 27 in [3] and Lemma 6.2 in $[10])$.

Now, the process of extending sets of wffs to maximal sets is required.

Definition 5.8 (Maximal sets). Let L be an Eb4-logic, $\Gamma$ is an L-maximal set of wffs iff $\Gamma \nvdash_{L}^{d} \bar{\Gamma}(\bar{\Gamma}$ is the complement of $\Gamma$ ).

Lemma 5.9 (Extension to maximal sets). Let $L$ be an Eb4-logic closed under no other rules than those specified in Definition 5.4, $\Gamma$ and $\Theta$ sets of wffs such that $\Gamma \nvdash_{L}^{d} \Theta$. Then, there are sets of wffs $\Gamma^{\prime}$ and $\Theta^{\prime}$ such that $\Gamma \subseteq \Gamma^{\prime}, \Theta \subseteq \Theta^{\prime}, \Theta^{\prime}=\overline{\Gamma^{\prime}}$ and $\Gamma^{\prime} \nvdash_{L}^{d} \Theta^{\prime}$ (i.e., $\Gamma^{\prime}$ is an L-maximal set such that $\left.\Gamma^{\prime} \nvdash_{L}^{d} \bar{\Theta}^{\prime}\right)$.

Proof: Cf. Lemma 9 in [3] and Lemma 6.4 in [10].

Finally, Primeness Lemma should be proved.

Lemma 5.10 (Primeness). Let $L$ be an Eb4-logic closed under no other rules than those specified in Definition 5.4. If $\Gamma$ is an L-maximal set, then it is a fully regular prime L-theory.

Proof: This Lemma was already provided for the exact same frame of logics in [6, Lemma 7.5].

\section{Preliminary lemmas to the completeness theorem}

Following Routley et al. (cf. [11, Chapter 4]), a series of preliminary lemmas will be proved in order to be used in the completeness proofs for the Lt $i$-logics. We start by presenting the notion of a $\mathcal{T}$-theory. 
Definition 6.1 ( $\mathcal{T}$-theory). Let L be an Eb4-logic and $\mathcal{T}$ a fully regular prime L-theory (cf. Definition 5.1). A $\mathcal{T}$-theory is a set of formulas closed under Adjunction (Adj.) and $\mathcal{T}$-entailment $(\mathcal{T}$-ent.). That is, $a$ is a $\mathcal{T}$-theory if whenever $A, B \in a$, then $A \wedge B \in a$; and if whenever $A \rightarrow B \in \mathcal{T}$ and $A \in a$, then $B \in a$.

Given the fact that $\mathcal{T}$ is regular (i.e., if $\vdash_{E b 4} A \rightarrow B$, then $A \rightarrow B \in \mathcal{T}$ ), it is remarkable that any $\mathcal{T}$-theory is an Eb4-theory. Therefore, if $\vdash_{L} A \rightarrow$ $B$ and $A \in a$, then $B \in a$, given that $a$ is closed under $\mathcal{T}$-ent.

Now, some relations on sets of $\mathcal{T}$-theories will be displayed.

Definition 6.2 (The sets $K^{T}, K^{C}$ ). Let $\mathcal{T}$ be a fully regular and prime Eb4-theory. $K^{T}$ is the set of all $\mathcal{T}$-theories, and $K^{C}$ is the set of all a-consistent non-empty and prime $\mathcal{T}$-theories (cf. Definition 5.2).

Definition 6.3 (The relations $R^{T}, R^{C}$ and $\vDash^{C}$ ). Let $\mathcal{T}$ be a fully regular and prime $\mathcal{T}$-theory and $K^{T}$ and $K^{C}$ be defined as in Definition 6.2. $R^{T}$ is defined on $K^{T}$ as follows: for all $a, b, c \in K^{T}, R^{T} a b c$ iff for all wffs $A$, $B,(A \rightarrow B \in a \& A \in b) \Rightarrow B \in c$. Next, $R^{C}$ is the restriction of $R^{T}$ to $K^{C}$. On the other hand, $\vDash^{C}$ is defined as follows: for any $a \in K^{C}$ and wff $A, a \models^{C} A$ iff $A \in a$.

Next, we define a unary operator on $K^{C}$.

Definition 6.4 (The operation $*^{C}$ ). The unary operation $*^{C}$ is defined on $K^{C}$ as follows: for each $a \in K^{C}, a^{*^{C}}=\{A \mid \neg A \notin a\}$.

Let L be an Eb4-logic, we will use the Extension Lemma (cf. Lemma 5.9) to build a fully regular and prime L-theory $\mathcal{T}$ in Proposition 7.3. For now, we define upon $\mathcal{T}$ the notions of the sets and relations expressed above (Definitions 6.2-6.4) and we define the following notion.

Definition 6.5 (The canonical Eb4-model). Let L be an Eb4-logic, the canonical L-model is the structure $\left\langle\mathcal{T}, K^{C}, R^{C}, *^{C}, \vDash^{C}>\right.$, whose members are understood according to definitions 6.2-6.4.

The canonical Eb4-model will be shown to be an Eb4-model by means of which non-theorems of $\mathrm{L}$ are falsified. 
Now, some useful lemmas for the completeness theorem developed in Section 7 will be proved. Let us suppose that we are given a fully regular and prime Eb4-theory $\mathcal{T}$ upon which the items $K^{T}, K^{C}, R^{C}, *^{C}, \vDash^{C}$ are defined as in Definition 6.5. First, we investigate the relations $R^{T}$ and $R^{C}$. Lemma 6.6 (Defining $x$ for $a, b$ in $R^{T}$ ). Let $a, b$ be non-empty $\mathcal{T}$-theories. The set $x=\{B \mid \exists A(A \rightarrow B \in a \mathscr{G} A \in b)\}$ is a non-empty $\mathcal{T}$-theory such that $R^{T} a b x$.

Proof: $x$ is a $\mathcal{T}$-theory: by T2 and the fact that $x$ is closed under PREF. $x$ is non-empty: by A13 (cf. [9, Lemma 5.5]).

Lemma 6.7 (Extending $b$ in $R^{T} a b c$ to a member in $K^{C}$ ). Let $a$ and $b$ be non-empty $\mathcal{T}$-theories, and $a$ and $c$ a-consistent, prime $\mathcal{T}$-theories such that $R^{T} a b c$. Then, there is an a-consistent (and non-empty) prime $\mathcal{T}$-theory $x$ such that $b \subseteq x$ and $R^{T}$ axc.

Proof: By the Kuratowski-Zorn's Lemma and T8 and T9 (cf. [11, pp. 309, ff.] and [9, Lemma 5.6]).

Lemma 6.8 (Extending $a$ in $R^{T} a b c$ to a member in $K^{C}$ ). Let $a$ and $b$ be non-empty $\mathcal{T}$-theories and $c$ an a-consistent, prime $\mathcal{T}$-theory such that $R^{T} a b c$. Then, there is an a-consistent (and non-empty) prime $\mathcal{T}$-theory $x$ such that $a \subseteq x$ and $R^{T} x b c$.

Proof: Cf. [9, Lemma 5.7].

Next, we set a definition to consider in relation to the succeeding lemma, which shows that the relation $\leq^{C}$ is just a set inclusion relation among a-consistent and non-empty prime $\mathcal{T}$-theories.

Definition 6.9 (The relation $\leq^{C}$ ). For any $a, b \in K^{C}: a \leq^{C} b$ iff $R^{C} \mathcal{T} a b$. LEMma 6.10 ( $\leq^{C}$ and $\subseteq$ are coextensive). For any $a, b \in K^{C}: a \leq^{C} b$ iff $a \subseteq b$.

Proof: $(\Rightarrow)$ By A1. $\quad(\Leftarrow)$ By $\quad$ Definitions $6.2, \quad 6.3$ and 6.9 (cf. [9, Lemma 5.9]).

In relation to the later, we also set the following lemma.

Lemma 6.11 (Extension to prime $\mathcal{T}$-theories). Let a be a $\mathcal{T}$-theory and $A$ a wff such that $A \notin a$. Then, there is a prime $\mathcal{T}$-theory $x$ such that $a \subseteq x$ and $A \notin x$. 
Proof: By simply applying the Kuratowski-Zorn Lemma as in [11, Chapter 4, pp. 310-311].

Throughout the following lemmas, the unary operator $*$ will be investigated $^{5}$.

Lemma 6.12 (Primeness of $*$-images). Let a be a prime $\mathcal{T}$-theory. Then, (1) $a^{*^{C}}$ is a prime $\mathcal{T}$-theory as well; (2) for any wff $A, \neg A \in a^{*^{C}}$ iff $A \notin a$.

Proof: (1) $a^{*}$ is closed under $\mathcal{T}$-ent, by the fact that $\mathcal{T}$ is closed by $\mathrm{CON}$; $a^{*}$ is closed under Adj., by T6; $a^{*}$ is prime, by T7. (2) By A7 and A8.

Lemma $6.13\left(*^{C}\right.$ is an operation on $\left.K^{C}\right)$. Let a be an a-consistent and non-empty prime $\mathcal{T}$-theory, then $a^{*^{C}}$ is an a-consistent and non-empty $\mathcal{T}$-theory as well.

Proof: In Lemma 6.12, it was already proved that, given our hypothesis, $a^{*}$ is also a prime $\mathcal{T}$-theory. Next, it is also clear that $a^{*}$ is a-consistent: there is some wff $A$ such that $A \in a$ ( $a$ is non-empty); therefore, $\neg A \notin a^{*}$ by Lemma 6.12 . Similarly, since $a$ is a-consistent, there is some wff $A$ such that $A \notin a$; therefore, $\neg A \in a^{*}$ by the same lemma.

Finally, next lemma proves that the relation $\vDash^{C}$ follows the clauses (i)-(v) in the definition of an Eb4-model (cf. Definition 4.1).

Lemma 6.14 (The relation $\vDash^{C}$ and clauses (i)-(v)). For all $a, b, c \in K^{C}$ and wffs $A, B$ :

(i) $\left(a \leq^{C} b\right.$ and $\left.a \vDash^{C} p\right) \Rightarrow b \vDash^{C} p$

(ii) $a \vDash^{C} A \wedge B$ iff $a \vDash^{C} A$ and $a \vDash^{C} B$

(iii) $a \vDash^{C} A \vee B$ iff $a \vDash^{C} A$ or $a \vDash^{C} B$

(iv) $a \vDash^{C} A \rightarrow B$ iff for all $b, c \in K^{C},\left(R^{C} a b c\right.$ and $\left.b \vDash^{C} A\right) \Rightarrow c \vDash^{C} B$

(v) $a \vDash^{C} \neg A$ iff $a^{*} \not \models^{C} A$

Proof: (i) Immediate by Lemma 6.10. (ii) By A2 and the fact that $a$ is closed under Adj. (iii) By A4 and primeness of $a$. (iv) $(\Rightarrow)$ Immediate by Definition 6.3; $(\Leftarrow)$ By contraposition, we suppose $a \not \nvdash^{C} A \rightarrow B$ and

${ }^{5}$ The label $C$ on $*^{C}$ will be omitted throughout the proofs of the following lemmas. 
show that there are $b, c \in K^{C}$ such that $R^{C} a b c, A \in b$ and $B \notin c$. We need Lemmas 6.6, 6.7 and 6.8. This kind of proof is already available in the literature (cf. [9, Lemma 5.13]). Finally, (v) is immediate by Definition 6.4.

\section{Completeness of $\mathrm{b} 4$}

In this section, we shall prove strong completeness of b4 w.r.t. the semantics defined in Section 4. We start by defining the useful concept of "set of consequences of a set of wffs".

Definition 7.1 (The set of consequences of $\Gamma$ in b4). The set of consequences in b4 of a set of wffs $\Gamma$ (in symbols $C n \Gamma[b 4]$ ) is defined as follows: $C n \Gamma[b 4]=\left\{A \mid \Gamma \vdash_{b 4} A\right\}$.

We note the following remark.

Remark 7.2 (The set of consequences of $\Gamma$ in b4 is a fully regular theory). It is obvious that for any $\Gamma, C n \Gamma[b 4]$ contains all theorems of $\mathrm{b} 4$ and is closed under the rules of b4. Consequently, it is also closed under b4-entailment.

Proposition 7.3 (The building of $\mathcal{T}$ ). Let $\Gamma$ be a set of wffs and $A$ a wff such that $\Gamma \nvdash_{b 4} A$. Then, there is a fully regular, a-consistent and prime b4-theory $\mathcal{T}$ such that $\Gamma \subseteq \mathcal{T}$ and $A \notin \mathcal{T}$.

Proof: Suppose $\Gamma \nvdash_{b 4} A$ (i.e., $A \notin C n \Gamma[b 4]$ given Remark 7.2). Then, $C n \Gamma[b 4] \nvdash_{b 4}^{d}\{A\}$ by Definition 5.6; otherwise $\left(B_{1} \wedge \ldots \wedge B_{n}\right) \vdash_{b 4} A$ for some $B_{1}, \ldots, B_{n} \in \Gamma$ and hence $A$ would be in $C n \Gamma[b 4]$ after all. Next, there is some (fully regular and a-consistent) prime b4-theory $\mathcal{T}$ such that $\Gamma \subseteq \mathcal{T}$ (since $\Gamma \subseteq C n \Gamma[b 4]$ ) and $A \notin \mathcal{T}$, by application of Lemmas 5.7 and 5.9.

Definition 7.4 (The canonical b4-model). The canonical b4-model is the structure $<\mathcal{T}, K^{C}, R^{C}, *^{C}, \vDash^{C}>$, where $K^{C}, R^{C}, *^{C}, \vDash^{C}$ are defined upon the b4-theory $\mathcal{T}$ as indicated in Definitions 6.2-6.4.

Once proved that the canonical b4-model is a b4-model, Proposition 7.3 is used to show $\Gamma \not \nvdash^{C} A$ in the canonical b4-model, this is, for any set of wffs $\Gamma$ and wff $A$ such that $\Gamma \nvdash_{b 4} A$, it will be shown that $A$ is not a semantic b4-consequence of $\Gamma$ (cf. Definition 4.5). 
In the following paragraphs, it will be proved that the canonical b4model is indeed a b4-model. Firstly, we prove that b4 postulates hold canonically by using a correspondent axiom scheme or rule from Definition 3.1. In this sense, we shall talk about corresponding postulate (c.p.) to a rule or axiom scheme.

Lemma 7.5 (b4 postulates hold canonically). The semantical postulates (p1-p13) hold in the canonical b4-model.

Proof: Lemma 6.10 is used to simplify these proofs. p1, p2, p5, p6 and p7 are proved as in [11, Chapter 4] and p9 and p11 as in [9]. Also, in [11, p. 339], the c.p. to the (disjunctive) affixing rule is proved. The c.p. to the (disjunctive) sufixing and preffixing rules (i.e., p3 and p4) can be proved similarly. Let us now prove p8, p10, p12 and p13.

p8 $\left(R \mathcal{T}^{*} \mathcal{T}^{*}\right)$ holds in the canonical b4-model: Suppose that there are wffs $A, B$ such that (1) $A \rightarrow B \in \mathcal{T}^{*}$ (i.e., $\neg(A \rightarrow B) \notin \mathcal{T}$ ) and $A \in \mathcal{T}$. Then, given that $\mathcal{T}$ is closed under CTE, we get $(2) A \wedge \neg B \notin \mathcal{T}$. Therefore, (3) $A \notin \mathcal{T}$ or $\neg B \notin \mathcal{T}$ since $\mathcal{T}$ is also closed under Adj. Finally, we get $\neg B \notin \mathcal{T}$ (i.e., $B \in \mathcal{T}^{*}$ ), given 1 and 3 .

p10 $R a b c \Rightarrow\left(a \leq c\right.$ or $\left.a^{*} \leq c\right)$ holds in the canonical b4-model: Suppose that there are $a, b, c \in K^{\bar{C}}$ and wffs $A, B$ such that (1) Rabc but (2) $A \in a, A \notin c, B \in a^{*}$ (i.e., $\neg B \notin a$ ) and $B \notin c$. Now, let $C$ be a wff such that (3) $C \in b$ ( $b$ was non-empty). On the other hand, we have (4) $A \vee B \in a$ by $2, \mathrm{~A} 4(A \rightarrow(A \vee B))$ and the fact that $a$ is closed under $\mathcal{T}$-ent. Similarly, by A10 in the form $(A \vee B) \rightarrow . \neg(A \vee B) \vee[C \rightarrow(A \vee B)]$, we get $(5) \neg(A \vee B) \vee[C \rightarrow(A \vee B)] \in a$. Therefore, $(6) \neg(A \vee B) \in a$ or $C \rightarrow(A \vee B) \in a$ ( $a$ is prime). If we suppose $C \rightarrow(A \vee B) \in a$, we get $A \vee B \in c$-given 1 and $3-$, contradicting $2(A \notin c$ and $B \notin c)$. Therefore, let us take $\neg(A \vee B) \in a$. Then, (7) $\neg A \wedge \neg B \in a$ by T7 $(\neg(A \vee B) \rightarrow(\neg A \wedge \neg B))$ and $(8) \neg B \in a$ by A2 $((\neg A \wedge \neg B) \rightarrow \neg B)$. However, 8 contradicts $2(\neg B \notin a)$.

$\mathrm{p} 12\left(R T a b \& R^{2} T c d e\right) \Rightarrow\left(a \leq c^{*}\right.$ or $d \leq c^{*}$ or $c \leq b$ or $\left.c \leq e\right)$ holds in the canonical b4-model: Suppose that there are $a, b, c, d, e \in K^{C}$ and wffs $A, B, C, D$ such that (1) $R \mathcal{T} a b$ and $R^{2} \mathcal{T}$ cde but (2) $A \in a$, $A \notin c^{*}$ (i.e., $\neg A \in c$ ) $, B \in d, B \notin c^{*}$ (i.e., $\neg B \in c$ ) $, C \in c, C \notin b, D \in c$ and $D \notin e$. By $\mathrm{A} 12$ in the form $[(A \vee B) \rightarrow(C \wedge D)] \vee\{[\neg(A \vee B) \wedge(C \wedge$ $D)] \rightarrow[(A \vee B) \rightarrow(C \wedge D)]\}$ and the fact that $\mathcal{T}$ is regular and prime, (3) $(A \vee B) \rightarrow(C \wedge D) \in \mathcal{T}$ or $[\neg(A \vee B) \wedge(C \wedge D)] \rightarrow[(A \vee B) \rightarrow(C \wedge D)] \in \mathcal{T}$ Let us suppose (4) $(A \vee B) \rightarrow(C \wedge D) \in \mathcal{T}$. Now, we have (5) $A \vee B \in a$ 
by $\mathrm{A} 4(A \rightarrow(A \vee B))$ and $2(A \in a)$. Thus, (6) $C \wedge D \in b$, given 4,5 and $R \mathcal{T} a b$ in 1 . However, $2(C \notin b)$ contradicts 6 . Let us now suppose (7) $[\neg(A \vee B) \wedge(C \wedge D)] \rightarrow[(A \vee B) \rightarrow(C \wedge D)] \in \mathcal{T}$. We have $(8) \neg A \wedge \neg B \in c$ by 2 and therefore, $(9) \neg(A \vee B) \in c$ given T7 (cf. Proposition 3.3). Thus, (10) $\neg(A \vee B) \wedge(C \wedge D) \in c$ by 2 . On the other hand, by d2 and 1 , there is (11) $x \in K^{C}$ such that $R \mathcal{T} c x$ and Rxde. Then, $(12)(A \vee B) \rightarrow(C \wedge D) \in x$ by 7 and 10 , since $R \mathcal{T} c x$ (by 11). Finally, (13) $C \wedge D \in e$ since we have 12, $R x d e$ (in 11) and $A \vee B \in d$ (by 2). However, 13 contradicts $D \notin e$ in 2.

$\mathrm{p} 13($ Rabc\& Rcde $) \Rightarrow\left(a \leq c\right.$ or $b \leq c$ or $c^{*} \leq c$ or $d \leq c$ or $\left.b \leq e\right)$ holds in the canonical b4-model: Suppose that there are $a, b, c, d, e \in K^{C}$ and wffs $A, B, C, D, E$ such that (1) Rabc \& Rcde but (2) $A \in a \&$ $A \notin c ; B \in b \& B \notin c ; C \in c^{*} \& C \notin c ; D \in d \& D \notin c ; E \in b$ $\& E \notin e$. Then, we have (3) $B \wedge E \in b$ and also (4) $(A \vee D) \vee C \in a$ by $\mathrm{A} 4$ (in the form $A \rightarrow[A \vee(D \vee C)]$ ) and T3. Now, by A13 in the form $[(A \vee D) \vee C] \rightarrow .(B \wedge E) \rightarrow\{[[((A \vee D) \vee C) \vee(B \wedge E)] \vee \neg[((A \vee$ $D) \vee C) \vee(B \wedge E)]] \vee[((A \vee D) \vee C) \rightarrow(B \wedge E)]\}$ and 4, we obtain (5) $(B \wedge E) \rightarrow\{[[((A \vee D) \vee C) \vee(B \wedge E)] \vee \neg[((A \vee D) \vee C) \vee(B \wedge E)]] \vee[((A \vee$ $D) \vee C) \rightarrow(B \wedge E)]\} \in a$. Then, $(6)\{[((A \vee D) \vee C) \vee(B \wedge E)] \vee \neg[((A \vee$ $D) \vee C) \vee(B \wedge E)]\} \vee[((A \vee D) \vee C) \rightarrow(B \wedge E)] \in c$, given Rabc in 1, 3 and 5. This is, $(7)\{[((A \vee D) \vee C) \vee(B \wedge E)] \vee \neg[((A \vee D) \vee C) \vee(B \wedge E)]\} \in c$ or $[((A \vee D) \vee C) \rightarrow(B \wedge E)] \in c$ since $c$ is prime. Let us suppose (8) $[((A \vee D) \vee C) \rightarrow(B \wedge E)] \in c$. Similarly as in 4 , we have $(9)(A \vee D) \vee C \in d$ by A4 and T3 given $2(D \in d)$. Then, (10) $B \wedge E \in e$ by 8 and 9 given $R c d e$ in 1 . However, 10 contradicts $2(E \notin e)$. Therefore, we suppose (11) $[((A \vee D) \vee C) \vee(B \wedge E)] \vee \neg[((A \vee D) \vee C) \vee(B \wedge E)] \in c$. Thus, by primeness of $c,[((A \vee D) \vee C) \vee(B \wedge E)] \in c$ or $\neg[((A \vee D) \vee C) \vee(B \wedge E)] \in c$. Suppose (12) $[((A \vee D) \vee C) \vee(B \wedge E)] \in c$, i.e., $(13)(A \vee D) \vee C \in c$ or $(B \wedge E) \in c$, given the fact that $c$ is prime. Now, we have (14) $(A \vee D) \vee C \notin c$ by 2 . Therefore, (15) $B \wedge E \in c$ (i.e., $B \in c$ and $E \in c)$. However, $2(B \notin c)$ contradicts 15 . Finally, suppose $(16) \neg[((A \vee D) \vee C) \vee(B \wedge E)] \in c$. Then, by $\mathrm{T} 7$ and the fact that $c$ is closed by Adj., $(17) \neg((A \vee D) \vee C) \in c \&$ $\neg(B \wedge E) \in c$. Now, again by T7, (18) $\neg(A \vee D) \in c \& \neg C \in c$. But this contradicts $2(\neg C \notin c)$.

Proposition 7.6 (The canonical b4-model is a b4-model). The canonical b4-model is indeed a b4-model. 
Proof: Given Definition 7.4 and Proposition 7.3, the proof follows by the fact that $*^{C}$ is an operation on $K^{C}$ (Lemma 6.13), the adequacy of the canonical clauses (Lemma 6.14) and the fact that postulates hold canonically (Lemma 7.5).

Theorem 7.7 (Strong completeness of b4). For any set of wffs $\Gamma$ and wff $A$ : if $\Gamma \vDash_{b 4} A$, then $\Gamma \vdash_{b 4} A$.

Proof: For some set of wffs $\Gamma$ and wff $A$, suppose $\Gamma \nvdash_{b 4} A$. By Proposition 7.3, there is a fully regular, a-consistent and prime b4-theory $\mathcal{T}$ such that $\Gamma \subseteq \mathcal{T}$ and $A \notin \mathcal{T}$. Then, following Definition 7.4, the canonical b4-model is defined upon $\mathcal{T}$ and is indeed a b4-model, given Proposition 7.6. Then, $\Gamma \not \nvdash^{C} A$ since $\mathcal{T} \models^{C} \Gamma$ but $\mathcal{T} \not \nvdash^{C} A$. Therefore, $\Gamma \not \nvdash_{b 4} A$ by Definition 4.5.

\section{Routley-Meyer ternary relational semantics for the Lt $i$-logics}

In the present section, we endow the Lti-logics with a Routley-Meyer ternary relational semantics. Given that a Routley-Meyer semantics for an Eb4-logic L is provided when L-models together with the notion of L-validity are defined (cf. Definition 4.6), the idea is to give a semantical postulate corresponding to each one of the axiom schemes A14-A30 in Definition 3.5. Then soundness and completeness theorems for extensions of the logic b4 with any of these schemes are immediate.

Next, I display the list of corresponding postulates (c.p.) to the axiom schemes of the Lt $i$-logics. In general, a postulate $p j(14 \leq j \leq 30)$ will be referred to as corresponding to an axiom scheme $A j$ iff (1) $A j$ is true in any Eb4-model $\mathrm{M}$ which contains $p j$ and (2) $p j$ is provable in any canonical Eb4-model where $A j$ is true.

p14 $R a b c \Rightarrow\left(R c^{*} a b^{*}\right.$ or $R c^{*} b a^{*}$ or $R c^{*} a a^{*}$ or $\left.R c^{*} b b^{*}\right)$ is the c.p. to $\mathrm{A} 14(A \wedge \neg B) \rightarrow[(A \wedge \neg B) \rightarrow \neg(A \rightarrow B)]$ B) $\stackrel{\mathrm{p} 15}{\rightarrow}$ A]

$\mathrm{p} 16\left(R T a b \& R a^{*} c d\right) \Rightarrow\left(T^{*} \leq d\right.$ or $\left.b^{*} \leq d\right)$ is the c.p. to A16 $\neg B \vee$ $[\neg(A \rightarrow B) \rightarrow \neg B]$ 
p17 Raaa is the c.p to $\mathrm{A} 17[A \wedge(A \rightarrow B)] \rightarrow B$

p18 $R a a^{*} a^{*}$ is the c.p. to $\mathrm{A} 18[(A \rightarrow B) \wedge \neg B] \rightarrow \neg A$

p19 $R a^{*} a a$ is the c.p. to $\mathrm{A} 19 A \rightarrow[B \vee \neg(A \rightarrow B)]$

p20 $R a^{*} a^{*} a^{*}$ is the c.p. to A20 $\neg B \rightarrow[\neg A \vee \neg(A \rightarrow B)]$

p21 $R a^{*} b c \Rightarrow\left(b \leq a\right.$ or $\left.b \leq a^{*}\right)$ is the c.p. to $\mathrm{A} 21[\neg(A \rightarrow B) \wedge \neg A] \rightarrow A$ $\mathrm{p} 22 R$
$(A \vee \neg B)$

p23 $R a^{*} b c \Rightarrow\left(a \leq c\right.$ or $\left.a^{*} \leq c\right)$ is the c.p. to $\mathrm{A} 23[\neg(A \rightarrow B) \wedge B] \rightarrow \neg B$

$\mathrm{p} 24\left(R a b c \& R b^{*} d e\right) \Rightarrow(a \leq e$ or $b \leq e$ or $d \leq c)$ is the c.p. to A24 $B \rightarrow\{[B \wedge \neg(A \rightarrow B)] \rightarrow A\}$

p25 $R T a b \Rightarrow R T^{*} a b$ is the c.p. to A25 $(A \rightarrow B) \vee \neg(A \rightarrow B)$

p26 $R T^{*} T^{*} T$ is the c.p. to A26 $(\neg A \vee B) \vee \neg(A \rightarrow B)$

p27 Raaa* or $R a^{*} a a^{*}$ is the c.p. to A27 $[(A \rightarrow B) \wedge(A \wedge \neg B)] \rightarrow$ $\neg(A \rightarrow B)$

p28 $R T a b \Rightarrow\left(R T^{*} a a^{*}\right.$ or $\left.R b^{*} a a^{*}\right)$ is the c.p. to $\mathrm{A} 28 \neg(A \rightarrow B) \vee[(A \wedge$ $\neg B) \rightarrow \neg(A \rightarrow B)]$

p29 $\left(R T a b \& R a^{*} c d\right) \Rightarrow\left(T^{*} \leq d \& b^{*} \leq d \& c \leq a^{*}\right)$ is the c.p. to A29 $\{[\neg(A \rightarrow B) \wedge \neg A] \rightarrow \neg B\} \vee \neg B$

p30 $\left(R T a b \& R a^{*} c d\right) \Rightarrow(c \leq T$ or $c \leq b$ or $a \leq d)$ is the c.p. to A30 $\{[\neg(A \rightarrow B) \wedge B] \rightarrow A\} \vee A$

Next, the notion of an Lt $i$-model $(1 \leq i \leq 8)$ is defined. 
Definition 8.1 (Lt $i$-models). An Lt $i$-model $(1 \leq i \leq 8) \mathrm{M}$ is a structure $<T, K, R, *, \vDash>$ where $K, T, R, *$ and $\vDash$ are defined according to the Definition 4.1 and $R$ is also subject to an additional set of postulates for each Lt $i$-logic:

Lt1-models: p14-p16;

Lt2-models: p17-p23;

Lt3-models: p14, p15, p18, p19, p22-p24;

Lt4-models: p16, p17, p20-p22;

Lt5-models: p17-p21, p23, p25-p27;

Lt6-models: p17, p20, p21, p23, p26, p28, p29;

Lt7-models: p14, p18, p19, p21, p23, p26, p30;

Lt8-models: p14, p21, p23, p26, p29, p30.

The definitions of truth, validity and semantic consequence in the Lt $i$ models are defined as in Definitions 4.3-4.5. Now, given that Lemmas and Propositions were already proved for Eb4-logics and that the Lt $i$-logics are indeed Eb4-logics, it suffices to prove that axioms A14-A30 are valid in Eb4-models where p14-p30 hold.

Proposition 8.2 (Validity of A14-A30). Let $\mathfrak{M}$ be a class of Eb4-models and $\mathrm{M} \in \mathfrak{M}$. Then, for any $j(14 \leq j \leq 30), A j$ is true in $\mathrm{M}$ iff $p j$ holds in M.

Proof: We proceed as in Proposition 4.9. A few instances will suffice as an illustration. In particular, we display the proofs for A14, A16, A20, A24 and A29. Proofs for A17 and A18 are already in the main literature [11, Chapter 4] and those for A25 and A27 can be found in [9, p. 13]. The rest of the axiom schemes of the Lt $i$-logics can be proved in a similar way.

(A14) $(A \wedge \neg B) \rightarrow[(A \wedge \neg B) \rightarrow \neg(A \rightarrow B)]$ is valid in any Eb4-model in which p14 holds: Suppose there are $a \in K$ in some Lti-model M and wffs $A, B$ such that $a \vDash A \wedge \neg B$ (i.e., (1) $a \vDash A$ and (2) $a^{*} \not \models B$ ) and (3) $a \not \models(A \wedge \neg B) \rightarrow \neg(A \rightarrow B)$. Then, we have for some $b, c \in K$, (4) $R a b c \&(5) b \vDash A \wedge \neg B$ (i.e., $b \vDash A$ and $\left.b^{*} \not \models B\right) \&(6) c \not \models \neg(A \rightarrow B)$ (i.e., $c^{*} \vDash A \rightarrow B$ ) by clause (iv) in Definition 4.1. Given 4 and p14 $\left(R a b c \Rightarrow\left(R c^{*} a b^{*}\right.\right.$ or $R c^{*} b a^{*}$ or $R c^{*} a a^{*}$ or $\left.R c^{*} b b^{*}\right)$ ), we get $(7) R c^{*} a b^{*}$ or 
$R c^{*} b a^{*}$ or $R c^{*} a a^{*}$ or $R c^{*} b b^{*}$. Now, whatever the case may be, we get a contradiction by applying clause (iv). If we suppose either (8) $R c^{*} a b^{*}$ or (9) $R c^{*} b b^{*}$, then given 6 and, respectively, 1 and 5 we shall get $(10) b^{*} \vDash B$, contradicting 5. Similarly, if we suppose either (11) $R c^{*} b a^{*}$ or (12) $R c^{*} a a^{*}$, we shall get (13) $a^{*} \vDash B$ again by 6 and, respectively, 5 and 1 . However, 2 and 13 are contradictory.

(A16) $\neg B \vee[\neg(A \rightarrow B) \rightarrow \neg B]$ is valid in any Eb4-model in which p16 holds: Suppose that in some Lt $i$-model $\mathrm{M}$ there are wffs $A, B$ such that (1) $T \not \models \neg B \vee[\neg(A \rightarrow B) \rightarrow \neg B]$, this is, (2) $T \not \models \neg B$ (i.e., $T^{*} \vDash B$ ) and (3) $T \not \models \neg(A \rightarrow B) \rightarrow \neg B$ given clause (iii). Now, by clause (iv), there are some $a, b \in K$ such that (4) $R T a b$, (5) $a \vDash \neg\left(A \rightarrow B\right.$ ) (i.e., $a^{*} \not \models A \rightarrow B$ ) and (6) $b \not \models \neg B$ (i.e., $b^{*} \vDash B$ ). Again by clause (iv) and 5 , there are $c, d \in K$ such that (7) $R a^{*} c d,(8) c \vDash A$ and (9) $d \not \models B$. Then, given 4,7 and p16 $\left(\left(R T a b \& R a^{*} c d\right) \Rightarrow\left(T^{*} \leq d\right.\right.$ or $\left.\left.b^{*} \leq d\right)\right)$, we get $(10) T^{*} \leq d$ or $b^{*} \leq d$. Now, given 9 , a contradiction follows from any case shown in 10 by applying the Hereditary Condition (Lemma 4.7) to 2 or 6 , respectively.

(A20) $\neg B \rightarrow[\neg A \vee \neg(A \rightarrow B)]$ is valid in any Lt $i$-model in which p20 holds: Suppose there are $a \in K$ in some Lt $i$-model $\mathrm{M}$ and wffs $A, B$ such that (1) $a \vDash \neg B$ (i.e., $a^{*} \not \models B$ ) and (2) $a \not \models \neg A \vee \neg(A \rightarrow B)$, this is, (3) $a \not k \neg A$ (i.e., $a^{*} \vDash A$ ) and (4) $a \not k \neg(A \rightarrow B)$ (i.e., $a^{*} \vDash A \rightarrow B$ ). Now, by p20 $\left(R a^{*} a^{*} a^{*}\right), 3$ and 4 , we get $(5) a^{*} \vDash B$, which contradicts 1 .

(A24) $B \rightarrow\{[B \wedge \neg(A \rightarrow B)] \rightarrow A\}$ is valid in any Eb4-model in which p24 holds: Suppose there are $a \in K$ in some Lt $i$-model M and wffs $A, B$ such that (1) $a \vDash B$ but (2) $a \not \models[B \wedge \neg(A \rightarrow B)] \rightarrow A$. Then, there are some $b, c \in K$ such that (3) Rabc, (4) $b \vDash B \wedge \neg(A \rightarrow B)$ (i.e., $b \vDash B$ and $b^{*} \not \models A \rightarrow B$ ) and (5) $c \not \models A$. Again, there are some $d, e \in K$ such that (6) $R b^{*} d e,(7) d \vDash A$ and (8) $e \not \models B$. Next, by 3, 6 and p24 ((Rabc \& $\left.R b^{*} d e\right) \Rightarrow(a \leq e$ or $b \leq e$ or $\left.d \leq c)\right)$, we get $(a \leq e$ or $b \leq e$ or $d \leq c)$. As in the previous proofs, whatever the case may be, a contradiction follows by the Hereditary Condition given $1,4,5,7$ and 8 .

(A29) $\{[\neg(A \rightarrow B) \wedge \neg A] \rightarrow \neg B\} \vee \neg B$ is valid in any Eb4-model in which p29 holds: Suppose that in some Lt $i$-model M there are wffs $A, B$ such that (1) $T \not \models \neg B \vee\{[\neg(A \rightarrow B) \wedge \neg A] \rightarrow \neg B\}$, this is, (2) $T \not \models \neg B$ (i.e., $T^{*} \vDash B$ ) and (3) $T \not \models[\neg(A \rightarrow B) \wedge \neg A] \rightarrow \neg B$. Then, by clause (iv), (4) $R T a b \&$ (5) $a \vDash \neg(A \rightarrow B) \wedge \neg A$ and (6) $b \not \models \neg B$ (i.e., $b^{*} \vDash B$ ) for $a$, $b \in K$. Now, given 5 , we have $(7) a \vDash \neg(A \rightarrow B)$ (i.e., $a^{*} \not \models A \rightarrow B$ ) and (8) $a \vDash \neg A$ (i.e., $a^{*} \not \models A$ ) by clause (ii). Again by applying clause (iv) to 7, there are $c, d \in K$ such that (9) $R a^{*} c d$, (10) $c \vDash A$ and (11) $d \not \models B$. 
Next, we have (12) $\left(T^{*} \leq d\right.$ or $b^{*} \leq d$ or $\left.c \leq a^{*}\right)$ given p29 ((RTab \& $\left.R a^{*} c d\right) \Rightarrow\left(T^{*} \leq d\right.$ or $b^{*} \leq d$ or $\left.\left.c \leq a^{*}\right)\right), 4$ and 9 . Let us suppose $T^{*} \leq d$ or $b^{*} \leq d$, then we have (13) $d \vDash B$-contradicting 11 - by applying the Hereditary Condition to either 2 or 6 , respectively. Finally, let us suppose $c \leq a^{*}$, then we get (14) $a^{*} \vDash A$ similarly, given 10 . However, 14 contradicts 8.

Next, we prove the adequacy of the semantical postulates.

Proposition 8.3 (Proof of p14-p30 in canonical Eb4-models). Let L be an Eb4-logic and for any $j(1 \leq j \leq 30)$ let the canonical $L \cup\{A j\}$-model $\mathrm{M}$ be a canonical Eb4-model. Then, $p j$ is provable in $\mathrm{M}$.

Proof: The proof proceeds as that of Lemma 7.5. Proofs for p17 and p18 on the one hand and p25 and p27 on the other hand can be found in [11, Chapter 4] and [9, p. 22], respectively. We prove Proposition 8.3 for the postulates used above in Proposition 8.2. The rest of the postulates can be proved similarly.

p14 $R a b c \Rightarrow\left(R c^{*} a b^{*}\right.$ or $R c^{*} b a^{*}$ or $R c^{*} a a^{*}$ or $\left.R c^{*} b b^{*}\right)$ holds in any canonical Eb4-model where A14 is valid. In order to prove p14, A14 in the following form will be used: $[(A \wedge E) \vee(C \wedge G)] \wedge \neg[(D \vee F) \wedge(B \vee$ $H)] \rightarrow \cdot\{[(A \wedge E) \vee(C \wedge G)] \wedge \neg[(D \vee F) \wedge(B \vee H)]\} \rightarrow \neg\{[(A \wedge E) \vee$ $(C \wedge G)] \rightarrow[(D \vee F) \wedge(B \vee H)]\}$. Suppose there are $a, b, c \in K^{C}$ and wffs $A, B, C, D, E, F, G, H$ such that (1) Rabc but (2) $A \rightarrow B \in c^{*}$, $A \in a, B \notin b^{*}$, (3) $C \rightarrow D \in c^{*}, C \in b, D \notin a^{*}$, (4) $E \rightarrow F \in c^{*}$, $E \in a, F \notin a^{*}$ and (5) $G \rightarrow H \in c^{*}, G \in b, H \notin b^{*}$. Given 2 and 4, we have $A \wedge E \in a$ and by A4, (6) $(A \wedge E) \vee(C \wedge G) \in a$. Similarly, given 2 and 3, we have $D \vee F \notin a^{*}$ (i.e., $\neg(D \vee F) \in a$ ) and by A4, (7) $\neg(D \vee F) \vee \neg(B \vee H) \in a$, this is, $(8) \neg[(D \vee F) \wedge(B \vee H)] \in a$ by applying T6. Next, we have $(9)[(A \wedge E) \vee(C \wedge G)] \wedge \neg[(D \vee F) \wedge(B \vee H)] \in a$ by 6 and 8. Now, by applying A14 in the aforementioned form, we get (10) $\{[(A \wedge E) \vee(C \wedge G)] \wedge \neg[(D \vee F) \wedge(B \vee H)]\} \rightarrow \neg\{[(A \wedge E) \vee(C \wedge G)] \rightarrow$ $[(D \vee F) \wedge(B \vee H)]\} \in a$. As before, given 2 and 4 , we have $C \wedge G \in b$ and by A4, (11) $(A \wedge E) \vee(C \wedge G) \in b$. Similarly, given 1 and 4 , we get $B \vee H \notin b^{*}$ (i.e., $\left.\neg(B \vee H) \in b\right)$ and by A4, (12) $\neg(D \vee F) \vee \neg(B \vee H) \in b$. Whence, $(13) \neg[(D \vee F) \wedge(B \vee H)] \in b$ by T6. Then, we get $(14)[(A \wedge E) \vee$ $(C \wedge G)] \wedge \neg[(D \vee F) \wedge(B \vee H)] \in b$ given 11 and 13. Now we have 1, 10 and 14, therefore $(15) \neg\{[(A \wedge E) \vee(C \wedge G)] \rightarrow[(D \vee F) \wedge(B \vee H)]\} \in c$ (i.e., $\left.[(A \wedge E) \vee(C \wedge G)] \rightarrow[(D \vee F) \wedge(B \vee H)] \notin c^{*}\right)$. In the following lines, we will 
get to $[(A \wedge E) \vee(C \wedge G)] \rightarrow[(D \vee F) \wedge(B \vee H)] \in c^{*}$, thus, a contradiction. By $2\left(A \rightarrow B \in c^{*}\right.$ and $\left.E \rightarrow F \in c^{*}\right)$ and T4, we get (16) $A \rightarrow(B \vee H) \in c^{*}$ and (17) $E \rightarrow(D \vee F) \in c^{*}$. Whence $(18)[A \rightarrow(B \vee H)] \wedge[E \rightarrow(D \vee F)] \in c^{*}$ and by T2, (19) $(A \wedge E) \rightarrow[(B \vee H) \wedge(D \vee F)] \in c^{*}$. Now, proceeding again as in 16-19, we get $(20)(C \wedge G) \rightarrow[(B \vee H) \wedge(D \vee F)] \in c^{*}$ given $2\left(C \rightarrow D \in c^{*}\right.$ and $\left.G \rightarrow H \in c^{*}\right)$. Finally, given 19 and 20, we get (21) $\{(A \wedge E) \rightarrow[(B \vee H) \wedge(D \vee F)]\} \wedge\{(C \wedge G) \rightarrow[(B \vee H) \wedge(D \vee F)]\} \in c^{*}$ and by applying T5, $(22)(A \wedge E) \vee(C \wedge G)] \rightarrow[(D \vee F) \wedge(B \vee H)] \in c^{*}$, as we needed.

p16 $\left(R \mathcal{T} a b \& R a^{*} c d\right) \Rightarrow\left(\mathcal{T}^{*} \leq d\right.$ or $\left.b^{*} \leq d\right)$ holds in any canonical Eb4-model where A16 is valid. Suppose there are $a, b, c \in K^{C}$ and wffs $A, B$ such that (1) RTab and $R a^{*} c d$ but (2) $A \in \mathcal{T}^{*}, A \notin d, B \in b^{*}$ and $B \notin d$. Then, we have $(3) \neg A \notin \mathcal{T}$, whence $(4) \neg(A \vee B) \notin \mathcal{T}$ by A2 and T7. Next, we have for an arbitrary wff $C,(5) \neg(A \vee B) \vee[\neg[C \rightarrow(A \vee B)] \rightarrow$ $\neg(A \vee B)] \in \mathcal{T}$ by A16. Therefore, $(6) \neg[C \rightarrow(A \vee B)] \rightarrow \neg(A \vee B) \in \mathcal{T}$ given 4 and the fact that $\mathcal{T}$ is prime. Now, we get $(7) \neg(A \vee B) \notin b$ by 2 ( $B \in b^{*}$, i.e., $\left.\neg B \notin b\right)$, A2 and T7. Lastly, given 1,6 and 7 , we have (8) $\neg[C \rightarrow(A \vee B)] \notin a$ (i.e., $\left.C \rightarrow(A \vee B) \in a^{*}\right)$ and on the other hand (9) $A \vee B \notin d$ by 2 . Thus, $C \notin c$ (by 1,9 and 8 ), contradicting the fact that $c$ is not empty.

p20 $R a^{*} a^{*} a^{*}$ holds in any canonical Eb4-model where A20 is valid. Suppose that there are $a \in K^{C}$ and wffs $A$ and $B$ such that (1) $A \rightarrow$ $B \in a^{*}(\neg(A \rightarrow B) \notin a)$ and $(2) A \in a^{*}$ (i.e., $\left.\neg A \notin a\right)$, whence (3) $\neg A \vee \neg(A \rightarrow B) \notin a$ since $a$ is prime. We have to prove $B \in a^{*}$. Then, by 3 and A20 $(\neg B \rightarrow[\neg A \vee \neg(A \rightarrow B)])$, we have (4) $\neg B \notin a$, this is, $B \in a^{*}$.

p24 $\left(R a b c \& R b^{*} d e\right) \Rightarrow(a \leq e$ or $b \leq e$ or $d \leq c)$ holds in any canonical Eb4-model where A24 is valid. Suppose there are $a, b, c, d, e \in K^{C}$ and wffs $A, B, C$ such that (1) $R a b c$ and $R b^{*} d e$ but (2) $A \in a, A \notin e, B \in b$, $B \notin e, C \in d$ and $C \notin c$. Then, (3) $A \vee B \in a$ by 2 and A4. Next, using A24 in the form $(A \vee B) \rightarrow .[(A \vee B) \wedge \neg[C \rightarrow(A \vee B)]] \rightarrow C$, we get (4) $[(A \vee B) \wedge \neg[C \rightarrow(A \vee B)]] \rightarrow C \in a$. Given 1, $2(C \notin c)$ and 4, we get (5) $(A \vee B) \wedge \neg[C \rightarrow(A \vee B)] \notin b$. Thus, $(6) A \vee B \notin b$ or $\neg[C \rightarrow(A \vee B)] \notin b$. However, given $2(B \in b)$ and A4, we clearly have $(7) \neg[C \rightarrow(A \vee B)] \notin b$ (i.e., $\left.C \rightarrow(A \vee B) \in b^{*}\right)$. Finally, (8) $A \vee B \in e$ (by 1,2 $(C \in d)$ and 7), contradicting $2(A \notin e$ and $B \notin e)$.

p29 $\left(R \mathcal{T} a b \& R a^{*} c d\right) \Rightarrow\left(\mathcal{T}^{*} \leq d\right.$ or $b^{*} \leq d$ or $\left.c \leq a^{*}\right)$ holds in any canonical Eb4-model where A29 is valid. Suppose there are $a, b, c, d \in K^{C}$ and wffs $A, B, C$ such that (1) $R \mathcal{T} a b$ and $R a^{*} c d$ but (2) $A \in \mathcal{T}^{*}, A \notin d$, 
$B \in b^{*}, B \notin d, C \in c$ and $C \notin a^{*}$. Given 2 and A4, we have (3) $A \vee B \in \mathcal{T}^{*}$ (i.e., $\neg(A \vee B) \notin \mathcal{T})$. Then, by A29 in the form $\neg(A \vee B) \vee\{[\neg[C \rightarrow(A \vee$ $B)] \wedge \neg C] \rightarrow \neg(A \vee B)\}$, we get $(4)[\neg[C \rightarrow(A \vee B)] \wedge \neg C] \rightarrow \neg(A \vee B) \in \mathcal{T}$ since $\mathcal{T}$ is regular and prime. As in 3, we have now (5) $A \vee B \in b^{*}$ (i.e., $\neg(A \vee B) \notin b)$ by A4 and 2. Next, we get $(6) \neg[C \rightarrow(A \vee B)] \wedge \neg C \notin a$ given 1, 4 and 5. Thus, $(7) \neg[C \rightarrow(A \vee B)] \notin a$ or $\neg C \notin a$ (i.e., $\left.C \in a^{*}\right)$. Therefore, $(8) \neg[C \rightarrow(A \vee B)] \notin a$ (i.e., $\left.C \rightarrow(A \vee B) \in a^{*}\right)$ given $2\left(C \notin a^{*}\right)$. Finally, we get (9) $A \vee B \in d$ (by 1, 2 and 8), contradicting 2 .

\section{Conclusion}

The variants of BN4 and E4 which contain Routley and Meyer's logic B were developed in [6] as possible alternatives to the systems BN4 and E4. The Lt $i$-logics are clearly related to the family of relevant logics since they enjoy the quasi relevance property characteristic of logics such as R-Mingle ${ }^{6}$. Given the position of Lti-logics among members in the family of relevant logics, the ternary relational semantics developed for them in the present paper could be seen as an essential tool to compare them to many other different logics of the said family. This work is also meant to be a detailing of how this kind of semantics works when it comes to 4-valued logics and an additional support to what is shown in [11, Chapter 4]: RoutleyMeyer semantics is a malleable and powerful instrument for interpreting non-classical logics.

Acknowledgements. Work supported by the Spanish Ministry of Education (FPU15/02651). Thanks are due to two anonymous referees of the BSL for useful comments on a previous version of this paper.

\section{References}

[1] A. R. Anderson, N. D. Belnap, Entailment: The Logic of Relevance and Necessity, vol. I, Princeton University Press (1975).

[2] A. R. Anderson, N. D. Belnap, J. M. Dunn, Entailment: The Logic of Relevance and Necessity, vol. II, Princeton University Press (1992).

${ }^{6}$ The reader can find the proof of this and other related properties in [6]. 
[3] R. T. Brady, Completeness proofs for the systems RM3 and BN4, Logique et Analyse, vol. 25 (1982), pp. 9-32.

[4] R. T. Brady (ed.), Relevant Logics and their Rivals, vol. II, Ashgate (2003).

[5] R. T. Brady, Universal Logic, CSLI (2006).

[6] S. M. López, Belnap-Dunn semantics for the variants of BN4 and E4 which contain Routley and Meyer's logic B, Logic and Logical Philosophy, (Online First), pp. 1-28, DOI: https://doi.org/10.12775/LLP.2021.004.

[7] R. K. Meyer, S. Giambrone, R. T. Brady, Where Gamma fails, Studia Logica, vol. 43 (1984), pp. 247-256, DOI: https://doi.org/10.1007/BF02429841.

[8] H. Omori, H. Wansing, 40 years of FDE: An Introductory Overview, Studia Logica, vol. 105 (2017), pp. 1021-1049, DOI: https://doi.org/10.1007/ s11225-017-9748-6.

[9] G. Robles, J. M. Blanco, S. M. López, J. R. Paradela, M. M. Recio, Relational Semantics for the 4-valued relevant logics BN4 and E4, Logic and Logical Philosophy, vol. 25(2) (2016), pp. 173-201, DOI: https://doi.org/10.12775/LLP.2016.006.

[10] G. Robles, J. M. Méndez, A companion to Brady's 4-valued relevant logic BN4: The 4-valued logic of entailment E4, Logic Journal of the IGLP, vol. 24(5) (2016), pp. 838-858, DOI: https://doi.org/10.1093/jigpal/jzw011.

[11] R. Routley, V. Plumwood, R. K. Meyer, R. T. Brady, Relevant Logics and their Rivals, vol. I, Ridgeview (1982).

[12] J. K. Slaney, Relevant Logic and Paraconsistency, [in:] L. Bertossi, A. Hunter, T. Schaub (eds.), Inconsistency Tolerance, vol. 3300 of Lecture Notes in Computer Science, Springer (2005), pp. 270-293, DOI: https://doi.org/10.1007/978-3-540-30597-2_9.

[13] R. Sylvan, V. Plumwood, Non-normal relevant logics, [in:] R. T. Brady (ed.), Relevant Logics and their Rivals, vol. II, Western Philosophy Series, Ashgate, Aldershot and Burlington (2003), pp. 10-16.

\section{Sandra M. López}

Universidad de Salamanca

Departamento de Filosofía, Lógica y Estética

37007, Campus Unamuno, Edificio FES

Salamanca, Spain

e-mail: sandralv@usal.es 